\title{
Intra-articular injection of Platelet rich plasma versus Hyaluronic acid for moderate knee osteoarthritis. A prospective, double-blind randomized controlled trial on 189 patients with follow-up for three years
}

\author{
Medhat Sdeek, Dina Sabry, Hisham El-Sdeek, Ahmed Darweash
}

From the Cairo University Hospital, Cairo, Egypt

Platelet-rich plasma injections have been proposed as an option for Conservative management of knee Osteoarthritis to provide symptomatic relief and also to delay the need for surgical intervention. Although almost all the current literatures provide some evidence on the benefits of this technique compared with Viscosupplementation, no studies have been performed to compare their Clinical outcomes. The purpose is to compare the Clinical outcomes provided by intraarticular injection of either Platelet rich plasma or Hyaluronic acid to treat knee Osteoarthritis.

Study Design: Randomized Controlled Trial

200 Patients with a history of Symptomatic knee Osteoarthritis (Kellgren-Lawrence grade 2 or 3 ) were randomized to undergo 3 blinded intra-articular injections of either Platelet rich plasma or Hyaluronic acid. The Interval between successive injections was 2 weeks. Patients were evaluated prospectively before the injection and then at 2, 6, 12, 24, 30 and 36 months. Evaluation was based on International Knee Documentation Committee (IKDC), Visual analog scale, VOMAC Score and the reinjection rate; 189 patients reached the final evaluation. Both platelet rich plasma and Hyaluronic acid were effective in improving knee Symptoms and functional status over time and remained stable over time up to 18 months Post-injection (No re-injection has been performed to any patient incorporated in this study during the first 18 months). The performed re-injections have been significantly lower in the PRP group. Both platelet rich plasma and Hyaluronic acid were effective in improving knee Symptoms and functional status over time and remained stable over time up to 18 months

No benefits or funds were received in support of this study. None of the authors have a conflict of interest.
Post-injection. The rate of the required re-injections has been significantly lower in platelet rich plasma group. platelet rich plasma provide longer duration of symptomatic relief, longer duration of functional status improvement and lesser number of needed re-injections than Hyaluronic acid when the patients have been followed through 36 months.

Keywords: Platelet-rich plasma; Hyaluronic acid; growth factors; intra-articular injections; osteoarthritis.

\section{INTRODUCTION}

Osteoarthritis is currently one of the most prevalent and morbid diseases around the world to

\footnotetext{
Medhat Sdeek ${ }^{1}, \mathrm{MD}$

Dina Sabry², PhD,

Hisham El-Sdeek ${ }^{3}$, MD

- Ahmed Darweash ${ }^{4}, \mathrm{MD}$

${ }^{1}$ Department of Orthopedics and Traumatology at Cairo University Hospital, Cairo, Egypt.

${ }^{2}$ Professor of Biochemistry at Faculty of Medicine, Cairo University, Cairo, Egypt.

${ }^{3}$ Department of Orthopedics and Traumatology at Al-Sahel Teaching Hospital.

${ }^{4}$ Department of Orthopedics and Traumatology at Faculty of Medicine, Suez University.

Correspondence : Dr. Medhat Sdeek, 9 Alhoria square, MaadiCairo, Egypt. Phone: 00201092094240.

Email : dr_medhat_sdeek@yahoo.com

- 2021, Acta Orthopædica Belgica.
} 
the degree that it is often considered in terms of organ failure (1). Moreover, osteoarthritis has a severe economic and social impact due to its effect on patient productivity and quality of life (2). Cartilage lacks the presence of vessels and nerves also it is isolated from the systemic regulation. This property of cartilage limits the regeneration ability of cartilage. Trauma, chronic overload, as well as metabolic and biological predisposition, may lead to an accelerated joint surface damage and eventually end-stage arthritis due to loss of tissue homeostasis (3). Numerous Protocols have been proposed as noninvasive treatment of osteoarthritis with different rates of success, but none has clearly shown an ability to alter the natural history of this disease, and therefore, none can be considered as an ideal treatment for osteoarthritis (4). Platelet rich plasma has been attracting attention as a promising procedure to stimulate repair or replace damaged cartilage. Alpha granules of platelets contain pools of growth factors which have been found to take part in the regulation of articular cartilage (5). Synovial hyaluronic acid is a high-molecular weight glycosaminoglycan that protects the cells and the intracellular collagen network from mechanical stress by acting as shock absorber. Platelet rich plasma consists of a volume of plasma containing platelets with high concentration (6). Hyaluronic acid when injected intra-articularly provides the Joint by the lost viscoelasticity (7). This study compares the biologic effect of Platelet rich plasma injection into the arthritic knee with the results obtained with another common injectable treatment. The hypothesis was that Platelet rich plasma could improve symptoms and function, possibly through the release of GFs and biologically active molecules, in patients affected by knee Osteoarthritis.

\section{PATIENT AND METHODS}

Between (September 2016 and September 2020) 200 Patients Have been enrolled in this double blind RCT after approval by the Hospital Ethics Committee and Scientific Board. Each patient has signed an Informed Consent form. The 200 Patients have been randomized into two groups; group (A) and group (B). Group-A received 3 injections of high molecular weight HA two Weeks intervals. Group-B received 3 injections of leucocyte poor platelet rich plasma two Weeks intervals. 200 Knees have been injected (No patients were treated bilaterally). The patients were selected according to the following inclusion criteria: age (45-65) years, History of chronic knee pain (at least 6 months), imaging findings suggesting degenerative knee disorder (KellgrenLawrence Score II and III), Primary Osteoarthritis, Ability to provide an informed Consent. the following Exclusion criteria had been applied: Age: $<45$ or $>65$; Kellgren-Lawrence score I or IV; Pretreatment visual analogue score less than 40, Rheumatoid arthritis, previous surgery or injection for the knee to be injected; severe mechanical axis deviation $\left(\mathrm{MAD}>10^{\circ}\right)$, Knee instability, knee OsteoChondral lesion hematological diseases, infections, immunodepression, patients on anticoagulants or antiplatelet therapy, hemoglobin level lower than 11 $\mathrm{g} / \mathrm{dL}$ or platelet count lower than $150,000 / \mathrm{mm}^{3}$. use of NSAIDs in the 5 days before blood donation. In order to keep the study blinded, blood harvesting has been performed in all patients but used only in platelet rich plasma group. For platelet rich plasma preparation, $20.0 \mathrm{ml}$ of autologous venous blood was drawn in Ambion ${ }^{\circledR}$ RNase-free conical tubes, using an aseptic technique from the antecubital area and mixed with $2 \mathrm{ml}$ of $0.129-\mathrm{mol} / \mathrm{L}$ sodium citrate. Double-centrifugation protocol has been followed in platelet rich plasma preparation. Sigma laboratory centrifuge has been used in Separation of the blood cell elements. The falcon tubes have been used. The first Span: At room temperature Centrifugation has been performed at $160 \mathrm{G}$ for 20 minutes. The first Span results in two basic components: in the upper fraction there is the Serum Component, while in the lower fraction there is the blood cell component. To increase the total amount of platelets collected for the second centrifugation, a sign was made $6-8 \mathrm{~mm}$ below the line that separated the basic component from the blood cell component, then all content above this point was drawn and transferred to another $5 \mathrm{ml}$ vacuum tube without anticoagulant. The second Span: At room temperature Centrifugation has been performed again at $400 \mathrm{G}$ for 15 minutes. The second span results in two components: SERUM Component and platelet rich plasma. 
Table I. - Patients and methods

\begin{tabular}{|l|c|c|}
\hline & PRP group & HA group \\
\hline Number & 95 & 54 \\
\hline Age (mean) & 60.2 & $78 \mathrm{~F}$ and $16 \mathrm{M}$ \\
\hline sex & $80 \mathrm{~F}, 15 \mathrm{M}$ & 27.1 \\
\hline BMI (mean) & 27.9 & 49 grade II, 45 grade III \\
\hline Degeneration level (K-L grade) & 43 grade II, 52 grade III & 3 injections, 2 weeks intervals \\
\hline Protocol of injection & 3 injections, 2 weeks intervals & $2.5 \mathrm{ml}$ \\
\hline Volume injected/ once & $2.5 \mathrm{ml}$ & \\
\hline
\end{tabular}

Approximately $3.0 \mathrm{ml}$ of platelet rich plasma was separated. The number of platelets in the isolated PRP fraction and also in the whole blood was assessed using the Sysmex Automated Hematology Analyzer. The mean platelet concentration of the whole blood was $324 \pm 25 \times 10^{3} / \mu 1$ (range: 277 $\left.356 \times 10^{3} / \mu 1\right)$. The mean platelet concentration of the PRP fraction was $2,664 \pm 970 \times 10^{3} / \mu 1$ (range: $\left.2,005-3,120 \times 10^{3} / \mu 1\right)$. This represents an 8.2 fold increase over the concentration in the whole blood. INJECTION: to prevent patients from discovering the substance they were receiving, the syringe was appropriately covered before the injection. After the injection, patients were sent home with instructions to restrict the use of the leg for Three days Postinjection and to use cold foments for one day postinjection (10 minutes/ hour). We have prescribed $10 \mathrm{mg} / \mathrm{kg}$ acetaminophen twice daily for three days post-injection, during this period, the use of nonsteroidal medication was forbidden. Patients were prospectively evaluated basally and at $2,6,12,18$, 24,30 and 36 months from the $3^{\text {rd }}$ injection using International Knee Documentation Committee (IKDC), Visual analog scale, VOMAC Score. Furthermore, adverse events, patient satisfaction and the re-injection rate were also recorded. In order to keep the study double blinded, all the clinical evaluations were performed by a staff member not involved in the injection procedure. No statistically significant differences were found between platelet rich plasma and Hyaluronic acid groups regarding age, sex, BMI, degeneration level, protocol of injection and the volume injected (Table I).

\section{RESULTS}

Results of the Visual Analogue scale revealed the following mean values in the PRP group (57.8 at the basal evaluation to 55.6 at 2 months, 53.6 at 6 months, 47.1 at 12 months, 40.2 at 18 months, 36.9 at 24 months, 37.8 at 30 months and 40.9 at 36 months). While revealed the following mean values in the HA group (59.3 at the basal evaluation to 57.9 at 2 months, 50.1 at 6 months, 50.3 at 12 months, 51.9 at 18 months, 53.6 at 24 months, 56.8 at 30 months and 60.3 at 36 months) (Fig. 1).

Results of The IKDC subjective score revealed the following mean values in the Platelet rich plasma group (49.1 at the basal evaluation to 60.8 at 2 months, 66.3 at 6 months, 67.9 at 12 months, 67.7 at 18 months, 63.6 at 24 months, 61.8 at 30 months and 55.2 at 36 months). While revealed the following mean values in the Hyaluronic acid group (47.3 at the basal evaluation to 62.4 at 2 months, 61.5 at 6 months, 61.6 at 12 months, 58.2 at 18

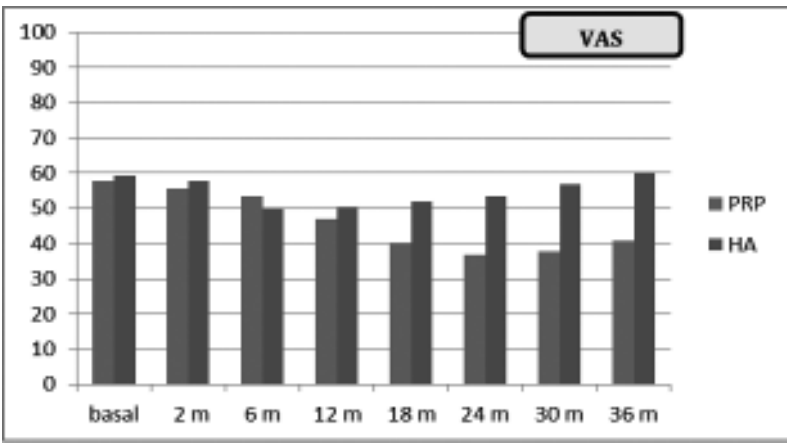

Fig. 1. - VAS. 


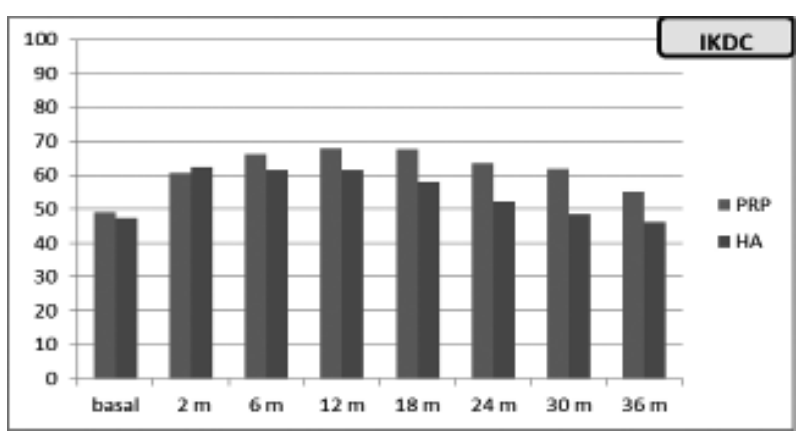

Fig. 2. - IKDC.

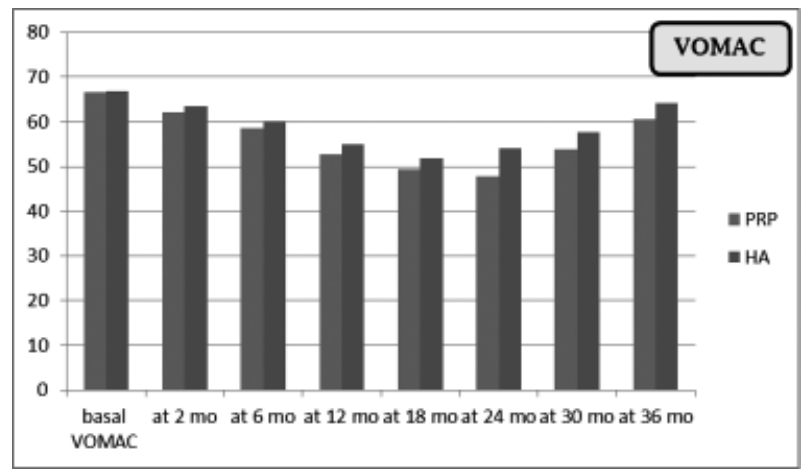

Fig. 3. - VOMAC.

months, 52.3 at 24 months, 48.4 at 30 months and 46.1 at 36 months) (Fig. 2).

Results of The VOMAC total score revealed the following mean values in the PRP group (66.5 at the basal evaluation to 62.2 at 2 months, 58.5 at 6 months, 52.8 at 12 months, 49.4 at 18 months, 47.7 at 24 months, 53.9 at 30 months and 60.6 at 36 months). While revealed the following mean values in the HA group (66.9 at the basal evaluation to 63.4 at 2 months, 60 at 6 months, 54.9 at 12 months, 51.9 at 18 months, 54 at 24 months, 57.6 at 30 months and 64.2 at 36 months) (Fig. 3).

Re-injection rate: In the platelet rich plasma group no re-injection has been performed during the first eighteen months. only two patients have been reinjected at the Twenty-four months follow up, five patients have been re-injected at the thirty months follow up and seven patients have been re-injected at the thirty-six months follow up. In the Hyaluronic acid group no re-injection has been performed during the first eighteen months. Six patients have been re-injected at the Twenty-four months follow

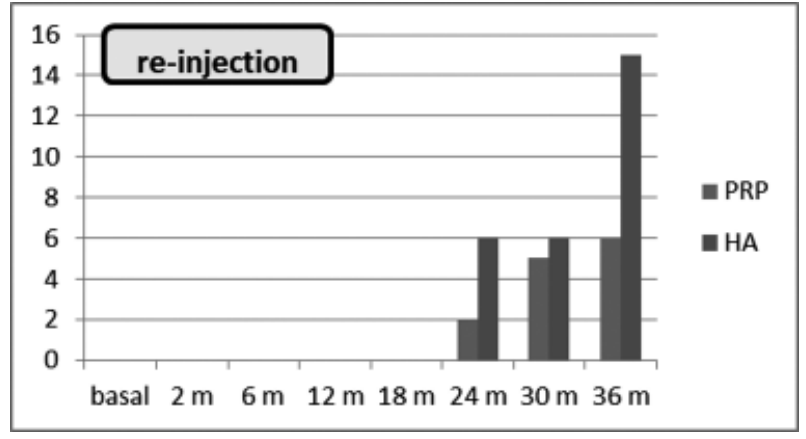

Fig. 4. - Re-injection rate.

up, six patients have been re-injected at the thirty months follow up and fifteen patients have been reinjected at the thirty-six months follow up. (Fig. 4)

Regarding the Complications; No major complications related to the injections were reported during neither treatment nor follow-up period. Worth mentioning that post-injection pain reaction was significantly higher in the platelet rich plasma group although this reaction was self-limiting and subsided within few days without compromising the overall outcome.

\section{DISCUSSION}

Due to the rising life expectancy and physical activity of the population, the prevalence of knee osteoarthritis has increased significantly (8). as a result; intra-articular injections such as platelet rich plasma and Hyaluronic acid have gained significant interest. The design of this study as a prospective double-blind randomized comparative study with three years of follow-up and applied on such a high number of patients make this study as one of the most available evidence on PRP application for knee osteoarthritis. This study has got over most of the weak points in the previous studies such as short term follow-up, small number of patient, being retrospective or absence of the comparative group. The results proved that Platelet rich plasma is superior to high molecular weight Hyaluronic acid when injected for treatment of knee osteoarthritis and we refer this to the BIOLOGICAL effects of Platelet rich plasma. The results of our study revealed that in Platelet rich plasma group, visual 
analogue score has improved up to two years of follow-up. While in Hyaluronic acid group, visual analogue score has improved only during the first year of follow-up. Moreover; at 36 months followup the VAS was much better in platelet rich plasma group (40.9) with comparison to the Hyaluronic acid group (60.3). The results of our study revealed that in Platelet rich plasma group, IKDC has improved up to 18 months of follow-up. While in Hyaluronic acid group, IKDC has improved only during the first year of follow-up. Moreover; at 36 months followup the IKDC was much better in platelet rich plasma group (55.2) with comparison to the Hyaluronic acid group (46.1). The results of our study revealed that in Platelet rich plasma group, VOMAC total score has improved up to two years of follow-up. While in Hyaluronic acid group, visual analogue score has improved only during the first 18 months of followup. Moreover; at 36 months follow-up the VOMAC total score was better in platelet rich plasma group (60.6) with comparison to the Hyaluronic acid group (64.2). The results of our study revealed that the reinjection rate was significantly less in the platelet rich plasma group. Many studies demonstrate Platelet rich plasma to be more effective than Hyaluronic acid (9). These studies have attributed the improved outcomes with Platelet rich plasma to its autologous makeup and high concentration of growth factors (10). A recent systematic review and meta-analysis of Randomized Controlled Trials that support the results of our study has been published. ${ }^{11}$ the safety and the significant clinical improvement of this procedure were confirmed. A higher Post-injection pain and swelling has been noticed in the Platelet rich plasma group. The results of this study confirm that the injection of this concentration of platelet rich plasma has a clinical subjective improvement and is effective in treatment of knee osteoarthritis. These results suggest the broadening of the clinical application of platelet rich plasma in treatment of knee osteoarthritis. In 2011 Kon et al published the results of his study that compares platelet rich plasma with low molecular weight Hyaluronic acid and also high molecular weight Hyaluronic acid in 150 patients. Efficacy of the treatment has been evaluated using both the International Knee Documentation Committee questionnaire and the visual analogue scale questionnaire. The evaluation has been performed at the beginning of treatment, at two months and at six months later. After two and six months a notable improvement has been observed in the three groups including all parameters. Assessment of Patient satisfaction in the platelet rich plasma group has revealed that it was more than in the other two groups. Unlike the other two groups, patients in platelet rich plasma group have an ascending course of improvement between the second and the six months. After two months, low molecular weight Hyaluronic acid and platelet rich plasma groups have showed similar results which were superior to the results in high molecular weight Hyaluronic acid group. After six months platelet rich plasma group has showed better results than in the other two groups (12). In a metaanalysis published by Khoshbin et al in 2013 similar results have been concluded. The meta-analysis concluded that platelet rich plasma is superior in its effect to normal saline and Hyaluronic acid when applied for mild or moderate osteoarthritis (13). In another study published in 2014 by Chang et al comparing the effect of platelet rich plasma when injected intra-articularly for treatment of knee osteoarthritis to that of Hyaluronic acid. The study concluded that in patients with knee osteoarthritis, platelet rich plasma has better functional outcome that continued for at least twelve months. More and longer improvement has been noticed in Patients of the platelet rich plasma group than in patients of the Hyaluronic acid group. Patients with milder forms of knee osteoarthritis have better results than in patients with advanced knee osteoarthritis (14). In 2019 Di Martino A et al have compared the effect of platelet rich plasma injection with the effect of Hyaluronic acid injection for knee osteoarthritis. Their study was double-blind only during the first year of follow-up (15). The current study has overcome this limitation being double-blind during the whole three years of follow-up. Limitations in this study include the mid-term follow-up period and absence of the placebo, but we think that these limitations don't affect the added value from the study. 


\section{CONCLUSSION}

The results of our study support the midterm effectiveness of platelet rich plasma injection over Hyaluronic acid injection for relieving pain and stiffness and improving knee functions in moderate knee osteoarthritis.

\section{REFERENCES}

1. Belén Cuervo, Mónica Rubio, Ramón Cugat, et al. New Approaches in the Treatment of Osteoarthritis. Int Journal of Orthopaedics. 2015; 232(1): 210-218.

2. Haywood L, McWilliams DF, Pearson CI, et al. Inflammation and angiogenesis in osteoarthritis. Arthritis Rheum. 2003; 48: 2173-2177.

3. Buckwalter JA, Brown TD. Joint injury, repair, and remodeling: roles in post-traumatic osteoarthritis. Clin Orthop Relat Res. 2004; 423: 7-16.

4. Hochberg MC, Altman RD, Brandt KD, et al. Guidelines for the medical management of osteoarthritis. Part II. Osteoarthritis of the knee: American College of Rheumatology. Arthritis Rheum. 1995; 38(11): 1541-1546.

5. Ulrich-Vinther M, Maloney MD, Schwarz EM, Rosier R, O'Keefe RJ. Articular cartilage biology. J Am Acad Orthop Surg. 2003; 11: 421-430.

6. Pietrzak WS, Eppley BL. Platelet rich plasma: biology and new technology. J Craniofac Surg. 2007; 16(6): 10431054.

7. Bannuru RR, Natov NS, Dasi UR, Schmid CH, McAlindon TE. Therapeutic trajectory following intraarticular hyaluronic acid injection in knee osteoarthritis Meta-analysis. Osteoarthritis Cartilage. 2011; 19: 611619.
8. Blagojevic M, Jinks C, Jeffery A, Jordan KP. Risk factors for onset of osteoarthritis of the knee in older adults: a systematic review and meta-analysis. Osteoarthritis Cartilage. 2010; 18(1): 24-33.

9. Lin KY, Yang CC, Hsu CJ, Yeh ML, Renn JH. Intraarticular injection of platelet-rich plasma is superior to hyaluronic acid or saline solution in the treatment of mild to moderate knee osteoarthritis: a randomized, doubleblind, triple-parallel, placebo-controlled clinical trial. Arthroscopy. 2019; 35(1): 106-117.

10. Lisi C, Perotti C, Scudeller L, et al. Treatment of knee osteoarthritis: platelet-derived growth factors vs hyaluronic acid: a randomized controlled trial. Clin Rehabil. 2018; 32(3): 330-339.

11. Belk JW, Kraeutler MJ, Houck DA, et al. Platelet-Rich Plasma versus Hyaluronic Acid for Knee Osteoarthritis. A Systematic Review and Meta-analysis of Randomized Controlled Trials. The American Journal of Sports Medicine 1-12 DOI: $10.1177 / 0363546520909397$.

12. Kon E, Mandelbaum B, Buda R, et al. Platelet-rich plasma intra-articular injection versus hyaluronic acid viscosupplementation as treatments for cartilage pathology: from early degeneration to osteoarthritis. Arthroscopy 2011; 27(11): 1490-501.

13. Khoshbin A, Leroux T, Wasserstein D, et al. The efficacy of platelet-rich plasma in the treatment of symptomatic knee osteoarthritis: a systematic review with quantitative synthesis. Arthroscopy. 2013; 29(12): 2037-48.

14. Chang K. Comparative effectiveness of platelet-rich plasma injections for treating knee joint cartilage degenerative pathology: a systematic review and meta-analysis. Arch Phys Med Rehabil. 2014; 95: 562-75.

15. Di Martino A, Di Matteo B, Papio T, et al. PlateletRich Plasma versus Hyaluronic Acid Injections for the Treatment of Knee Osteoarthritis. Results at 5 Years of a Double Blind Randomized Controlled Trial. The American Journal of Sports Medicine 2019; 47(2): 347-354. 